\title{
Coordination of an Unmanned Vehicle with Active Suspension Over Extreme Terrain
}

\author{
Kenneth J. Waldron, Muhammad Abdallah \\ Department of Mechanical Engineering, Stanford University, Stanford, CA, USA 94305-4021
}

\begin{abstract}
Active suspension is now a well-tried technology in road vehicles. It has been installed on a HMMV and demonstrated to significantly improve performance in rough road conditions ${ }^{1}$. This capability presents an opportunity for improved mobility in off-road conditions. The challenge is to devise a means of translating the desired trajectory of the vehicle into commands to the suspension actuators and the traction motors in an optimal, or near optimal manner. In this paper we describe part of a software architecture that was developed to enable such performance from a six-wheeled vehicle with active suspension and independent wheel drives. The vehicle was a concept developed under the DARPA Unmanned Ground Combat Vehicle Program.
\end{abstract}

\section{INTRODUCTION}

It has been demonstrated that active suspension can be used to reduce impact and vibration experienced by the driver and payload of an off-road vehicle traversing rough roads, and thereby allow greater speed with less driver stress ${ }^{1}$. What has not been explored is whether the active suspension capability can be used to improve vehicle performance over extreme terrain. The work reported here was done in the context of a robotic vehicle in which driver stress is not an issue. It also allows use of extreme attitudes, and rapid changes of attitude that might not be acceptable in a vehicle carrying humans.

Given that the vehicle has an active suspension system, the question is how may it be used to improve performance in extreme terrain conditions? This becomes the problem of translating motion commands to the vehicle as a whole into force, position or rate commands to the wheel and suspension actuators, as illustrated in Figure 1.

Of course, there is another relevant issue, which is the source of the position or rate commands on the vehicle as a whole. In a conventional vehicle they come from the driver via the control interface. The project described here was a robotic vehicle. The overall software and control architecture is described in Waldron et $a l^{2}$. Briefly, the architecture used has three layers of which the upper layer generates action plans to be executed by the proprioceptive layer that is the subject of the present paper.

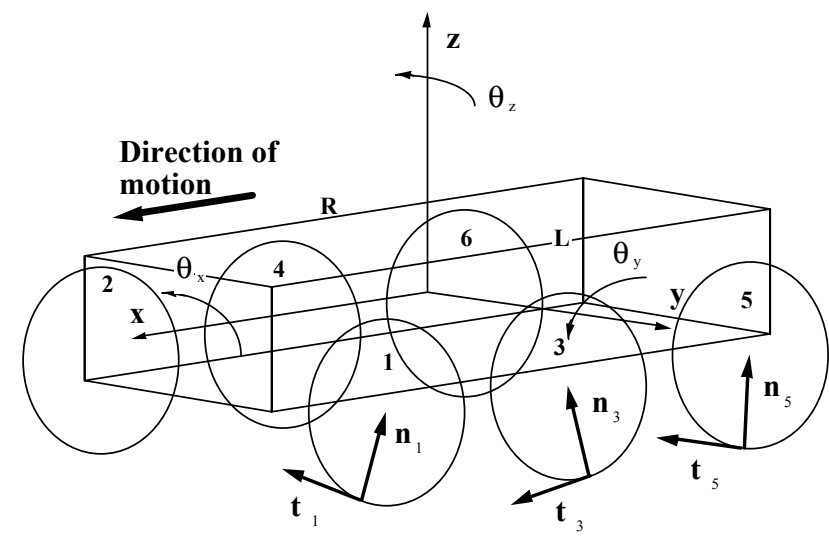

Figure 1: Vehicle coordinate system and motion degrees of freedom. The reference frame is aligned with the horizontal longitudinal axis of the body (x) and the vertical when the vehicle is resting on a level plane with the suspension positions neutral ( $\mathrm{z})$. Rotation about the $\mathrm{x}$ axis $\left(\theta_{\mathrm{x}}\right)$ is referred to as roll, about the $y$ axis $\left(\theta_{y}\right)$ is referred to as pitch, and about the $\mathrm{z}$ axis $\left(\theta_{\mathrm{z}}\right)$ is yaw. Because of the nonholonomic nature of the wheel-ground contact lateral (y) displacement, or velocity, cannot be directly controlled. The commandable degrees of freedom are, therefore, $\mathrm{x}, \mathrm{z}, \theta_{\mathrm{x}}, \theta_{\mathrm{y}}$, $\theta_{\mathrm{z}}$. In order to control vehicle configuration, suspension positions may be substituted for some of these degrees of freedom. 
The fundamental coordination principle that we attempt to implement in this work is minimization of the maximum ratio of tangential to normal contact force components among the wheel-ground contacts. The intended effect is to minimize the tendency toward slippage at any wheel. Of course, this assumes that the effective coefficients of friction at the wheel contacts are equal. A wheel contacting slippery mud may slip at a tangential to normal force ratio that is secure for the remaining wheels. Nevertheless since, as a practical matter, the soil properties at the wheel contacts are not a priori knowable, the above principle provides a serviceable compromise.

Although some of the principles presented here have broader applicability, we will restrict the discussion to vehicles with wheels arranged in two planes of operation that have individual drives for all wheels, and one actively controlled degree of freedom in each wheel suspension.

It is also assumed that sensors are installed to provide wheel torque and position or rate, two components of suspension force in the wheel plane, and suspension position. Likewise, an inertial measurement unit (IMU) mounted to the body of the vehicle with magnetometer and/or GPS correction of drift is assumed. Accelerometers mounted on the wheel hubs are also desirable to help correct for wheel dynamics.

\section{COMMANDED FORCE SYSTEM}

The commanded motions of the vehicle are compared to the actual motion of the vehicle body as measured by the IMU and used to generate a six axis rate error system that is then converted into a commanded acceleration system ${ }^{3}$. Multiplied into an inertia matrix this becomes an inertia force system that is combined with the vehicle weight to generate a six-axis commanded force system for the vehicle as a whole.

The force system acting on the body is decomposed into two planar force systems based on the wheel planes on either side of the vehicle. This is done in a manner similar to that described by Waldron $^{3}$. The solution is unique, subject to an assumption made about the distribution of lateral forces that cannot be controlled in this non-holonomic system. The details will not be repeated here.

The next task is to decompose each of the planar force systems into appropriate force and torque commands to the suspension and traction actuators, while attempting to at least approximate the minimax ratio of tangential to normal force principle. In order to do this it is first necessary to know the locations of the contacts between the wheels and the ground. Here we replace the actual contact, distributed over an area, by the point at which the line of action of the resultant contact force penetrates the tire. This point is what we refer to as the contact point. It is projected into the mid-plane of the wheel. The contact normal is assumed to lie along the line joining the contact point to the wheel axis in that plane. The normal force component is directed along that line directed from the contact to the axis. It may never be negative. That is, the contact only supports a compressive normal component. If a negative normal component is calculated it indicates that the vehicle is unstable and may overturn.

The tangential component of the contact force is, of course, directed normal to the contact normal through the point of contact in the wheel plane. 


\section{CONTACT POINT LOCATION}

There are actually two methods available for location of the contact point. The first is from the sensed wheel force data. Since we assume that two components of suspension force in the wheel plane, and wheel torque, are sensed, and the wheel weight, inertia and state of motion is known, it is possible to compute the line of action of the contact force. Thus, the contact point is identified by the intersection of that line with the tire (Figure 2). There are, in fact, two intersection points, but the correct one is, in principle, easily identified by applying the requirement that the normal component be positive.

The second approach is kinematic ${ }^{9}$, and depends on the ability to sense vehicle attitude and motion, and the suspension position. The position of the vehicle reference frame relative to a fixed frame is sensed by the IMU. The position of the suspension and wheel relative to the vehicle reference frame is sensed by resolvers, encoders, or other suitable position sensors. Application of the coordinate transformation between the vehicle frame and the fixed frame to these positions gives the positions of wheel and suspension relative to the fixed frame.

Likewise, we compute the six axis velocity of the vehicle body from IMU data, and transform into the fixed frame. The velocity of the suspension relative to the vehicle body, and the angular velocity of the wheel relative to the suspension are sensed and used to compute the velocity of the wheel axis, $\mathrm{O}$, and the angular velocity of the wheel relative to the fixed frame.

Since we have no control over velocity components normal to the wheel plane, we work with the velocity components in the wheel plane: $\mathrm{v}_{\mathrm{Ox}}, \mathrm{v}_{\mathrm{Oz}}$ and (using the coordinate system of Figure 1). $v_{O x}$ and $v_{O z}$ are the velocity components relative to a fixed reference frame of a point in the wheel plane that is fixed relative to the body coordinate frame. There is a unique point in the wheel, located in the wheel mid-plane, that is at rest relative to the fixed frame. That is the instantaneous center, I, of motion of the wheel shown in Figure 2. If the wheel is rolling without slipping on the ground, I will be located at the point of contact between wheel and ground. In general, it is not located at the point of contact, and its displacement from that point can be used to compute the slip of the wheel relative to the ground. The contact point lies at the intersection of the line IO with the wheel circumference, and the normal component of the contact force lies along the contact normal, IO.

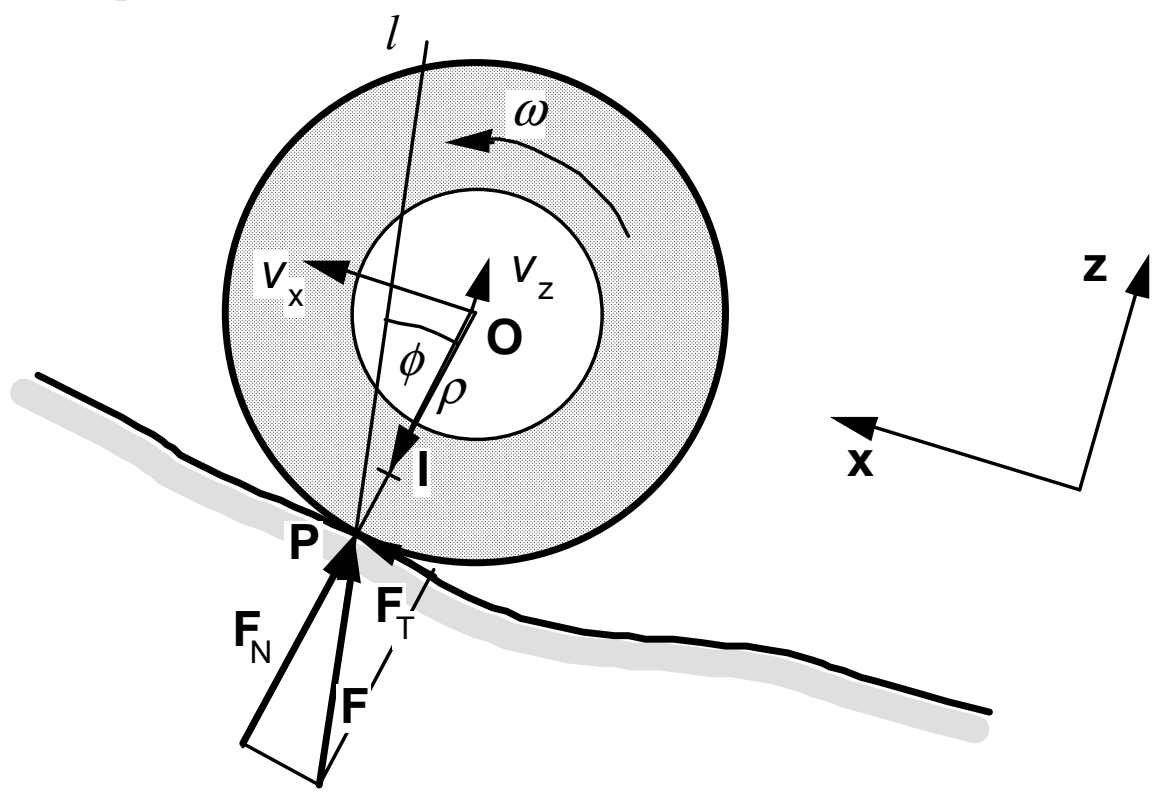

Figure 2: Wheel-ground contact geometry. The contact point, P, can be located either by identifying the line of action, $l$, of the contact force, and finding its intersection with the wheel circumference, or by locating the instantaneous center, I, of motion of the wheel relative to the ground. P lies at the intersection of line OI with the wheel circumference. 
The vector, $\rho$, that locates I from point $\mathrm{O}$ is found from

$$
\rho=\frac{\omega \times v_{\mathrm{O}}}{\omega^{2}} \text {. }
$$

The velocity, $v_{\mathrm{s}}$, of slip between the wheel and ground can then be calculated from

$$
v_{\mathrm{s}}=\omega \times(\mathbf{r}-\rho)
$$

where $\mathbf{r}$ is the vector from $\mathrm{O}$ to $\mathrm{P}$.

A fully three dimensional calculation is also possible, using the kinematic geometry of screw systems ${ }^{4}$. That formulation is more complex, but provides information on lateral slip, which may be useful.

\section{FORCE ALLOCATION}

It is now necessary to compute a set of contact forces at each of the wheels on each side of the vehicle that add up to the commanded resultant force. For the subject vehicle with six wheels, allocating the contact forces among the three wheels on one side of the vehicle is an underdetermined problem. This is also true for vehicles with eight, or more wheels. The methods described below are perfectly general and may be applied in those cases.

In the general case we attempt to apply the principle discussed above: minimizing the maximum ratio of the tangential to normal contact force components, to resolve the under-determinacy. There are a number of possible approaches to doing this, at least approximately. However, it is important to remember that whatever method is used the calculation must be performed in every update cycle, typically at greater than $100 \mathrm{~Hz}$, and the result must be usable as a control input.

Several different types of force allocation algorithm have been studied, or applied on past systems. It is possible, with relatively minor linearization, to formulate the spatial force allocation problem as a linear programming problem ${ }^{5,6}$. The resulting solutions are very near optimal, and this approach has been used as a baseline against which to measure the performances of alternative algorithms. When specialized to the planar system considered here no approximation is needed. The solution is optimal under the assumptions used. However, to the author's knowledge been implemented on a vehicle. The reasons are two. First the open, iterative character of the algorithm creates difficulties in the design of software that must run on uniform, rapid update cycles. Second, for linear programming problems of this dimension, the appropriate solution algorithm is the Simplex algorithm. It is the nature of this algorithm that the solution jumps from vertex to vertex of a polyhedral solution space. The resulting force commands exhibit discontinuities characterized as "chatter" which makes them undesirable as servo system commands.

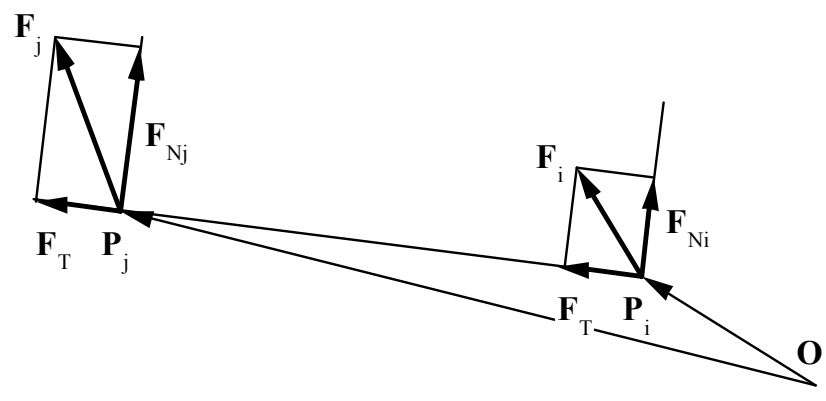

Figure 3: Geometric form of the zero interaction force condition. The components, $\mathbf{F}_{\mathrm{T}}$ of the contact forces at the points $\mathrm{P}_{\mathrm{i}}$ and $\mathrm{P}_{j}$ along the line $\mathrm{P}_{\mathrm{i}} \mathrm{P}_{\mathrm{j}}$ must be equal for every pair of contact points in the system. 
The Adaptive Suspension Vehicle $^{7}$, used an algorithm based on requiring that the interaction force between any pair of feet be zero. This algorithm was shown to be isomorphic with, but much more computationally efficient than, Moore-Penrose pseudoinversion of the system Jacobian $^{8}$. That is, it has the effect of minimizing the Euclidean Norm of the contact force magnitudes. Despite the fact that this was a legged system and we are presently discussing wheeled systems, the optimality criterion is the same,

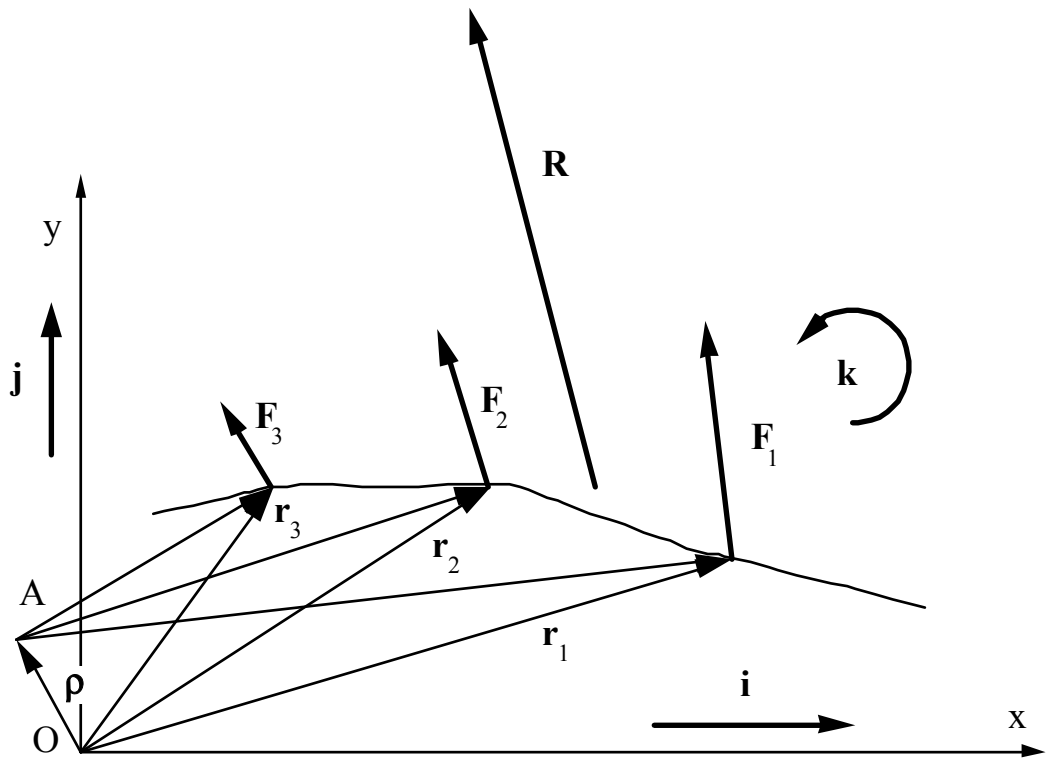

Figure 4: Force allocation using the zero interaction force principle. The magnitudes of the contact forces, $\mathbf{F}_{\mathrm{i}}$ are proportional to the distances of the contact points from a force center, A, and the contact forces are directed normal to the vectors from A to the contact points. alternatives.

Geometrically, the zero interaction force condition is as shown in Figure 3. It means that the components of the contact forces at any two wheels along the line joining the contact points are equal. Imposing this condition removes any tendency of pairs of wheels to work against each other. As has been demonstrated, it leads, via a straightforward geometric argument, to an elegantly simple set of equations that can be computed with great efficiency in real time (Kumar and Waldron, 1988).

Here the contact force, $\mathbf{F}_{\mathrm{i}}$, that must be generated at contact point $\mathrm{i}$ is given by the equation

$$
\mathbf{F}_{i}=S \mathbf{k} \times\left(\mathbf{r}_{i}-\rho\right)
$$

where, as shown in Figure 4, $\mathbf{r}_{\mathbf{i}}$ is the position, relative to the vehicle reference frame, of contact point $i, \rho$ is the position of point A, called the force center, and $S$ is the field intensity.

The vector, $\rho$, and the intensity, $S$ can be computed from the magnitude and line of action of the resultant force, $\mathbf{R}$, and the locations of the contact points via the following equations:

$$
S=\frac{T-|\overline{\mathbf{r}} \times \mathbf{R}|}{n I_{G}}, \quad \rho=\overline{\mathbf{r}}+\frac{\mathbf{k} \times \mathbf{R}}{n S}
$$

Here $T$ is the moment of the resultant force, $\mathbf{R}$, about the origin, $\overline{\mathbf{r}}$ is the location of the centroid of the $n$ contact points, and $I_{\mathrm{G}}$ is their moment of inertia about that centroid.

An alternative approach that can be applied to the present planar model is the simple principle that all contact forces are parallel, and parallel to the commanded resultant. The magnitudes of the contact forces are distributed linearly in proportion to their position normal to their direction, as shown in Figure 5. 


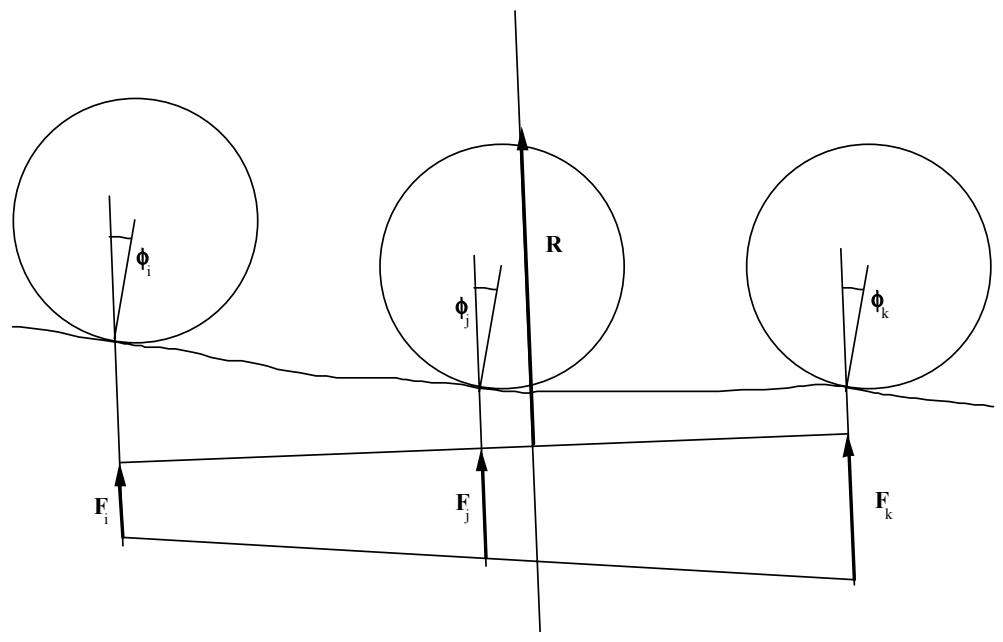

Figure 5: Linear contact force distribution produced by the zero interaction force condidtion applied to a planar force system.
Both the zero interaction force algorithm and the linear force distribution algorithm are, however, sub-optimal ${ }^{5}$ with respect to minimizing the ratio of tangential to normal force. They approach optimality, in this sense, in moderate terrain. Indeed, in cruise conditions the linear force distribution algorithm is optimal from a different viewpoint. It represents the most nearly uniform distribution of contact forces that yet sums to the commanded resultant. This is

important in the sense of minimizing the sinkage of the wheels into the soil, and hence minimizing the soil work and effective resistance, leading to greater range for given fuel weight. It is likewise, optimal in weak soil conditions in the sense of minimizing the tendency of contact failure.

In more extreme terrain both these criteria may perform poorly. The reason this happens is illustrated in Figure 6, which shows the mechanics of two locomotion elements in contact with a step obstacle: admittedly an extreme example. The example is the zero interaction force criterion, but a very similar argument can be applied to the linear force distribution criterion. Equalizing the components of the contact forces along the line joining the contact points in this case produces a very large ratio of tangential to normal force at one contact, with the traction forces at the two contacts actually being opposed to one another.

An algorithm based on the theory presented here, while restricted in the vehicle architectures to which it is applicable, much more nearly approaches optimality for locomotion in extreme terrain. The solution will be developed geometrically. However, the resulting equations, while quite different to those produced by the zero interaction force approach, are similarly simple and conducive to efficient, real-time solution.

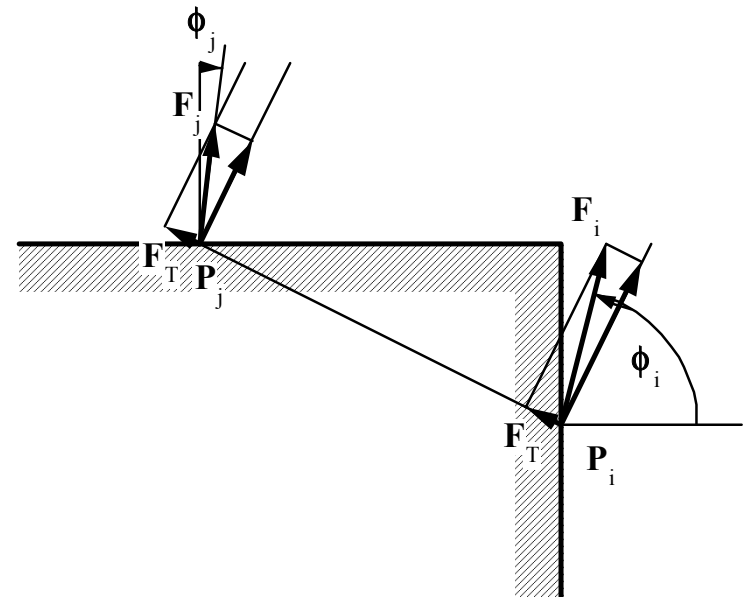

Figure 6: The zero interaction force condition applied to a pair of feet in contact with a step obstacle. The resulting contact forces are far from optimal. The friction angle at point $P_{i}$ is very large, and is actually opposed by that at point $\mathrm{P}_{\mathrm{j}}$. 


\section{TWO-POINT CONTACT}

When only two points are in contact the basic geometry is as shown in Figure 7. Since the forces $\mathbf{R}, \mathbf{F}_{\mathrm{i}}$ and $\mathbf{F}_{\mathrm{j}}$ form a three force set they must be concurrent. That is, the lines of action of $\mathbf{F}_{\mathrm{i}}$ and $\mathbf{F}_{\mathrm{j}}$ must intersect on the line of action of $\mathbf{R}$.

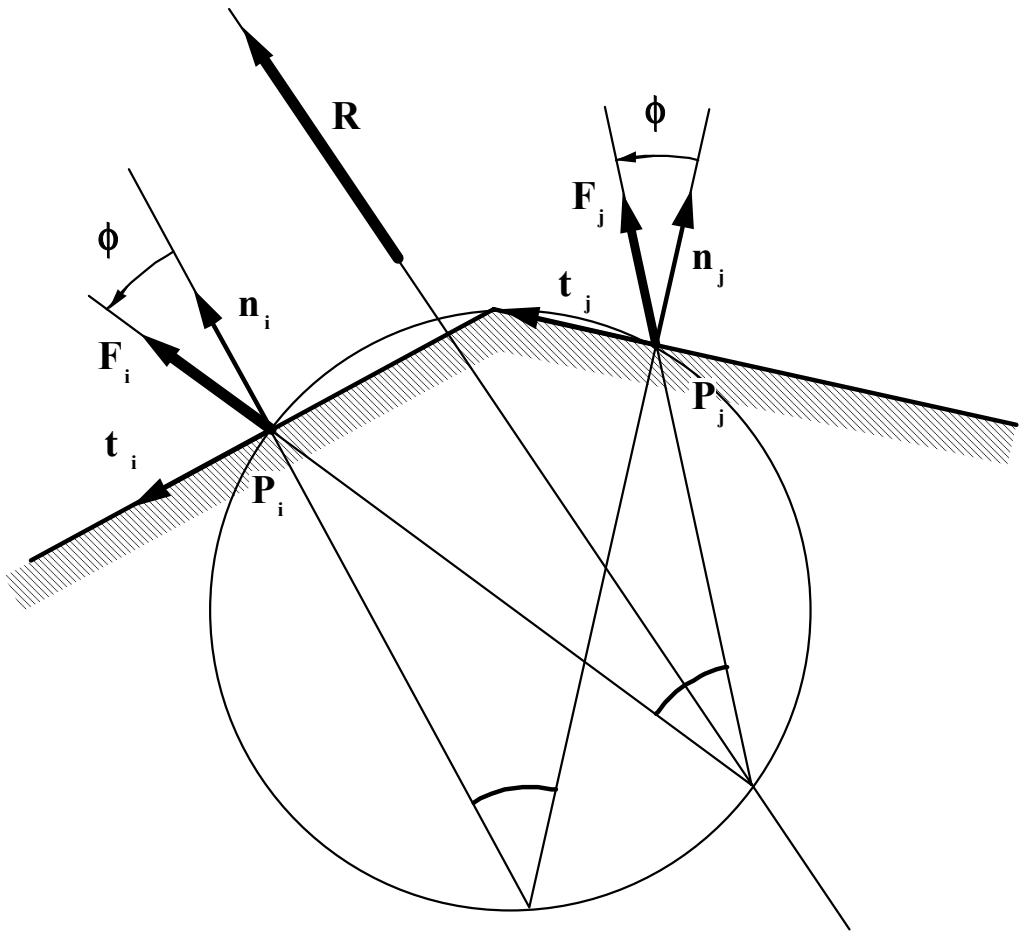

Figure 7: Geometric solution of the minimax problem for two contact points
If we draw the circle that passes through the two contact points and the intersection of the contact normals, as shown on Figure 7, the angle subtended at all points on the circumference of that circle by the line $\mathrm{P}_{1} \mathrm{P}_{2}$ is the same. This ensures that, if the lines of action of the contact forces intersect at the intersection of the line of action of the commanded force, $\mathbf{R}$, with this circle, the angles, $\phi$, are equal. Of course the tangent of $\phi$ is the ratio of the tangential to the normal contact force component. Thus, the criterion becomes minimizing the maximum value of $\phi$ over all the contacts.

If the concurrency point is moved outward along the line of action of $\mathbf{R}$, beyond the circle, the angle at one contact decreases, while that at the other increases. The same happens if the concurrency point is moved inward to the interior of the circle. Thus, the solution shown in Figure 7 is, in fact, the minimax solution, namely that solution that minimizes the maximum value of the friction angles.

\section{THREE OR MORE CONTACT POINTS}

In the case of vehicles with three or more wheels per side there will, in general, be more than two wheels in contact with the ground at any given time. This presents an even more underdetermined problem. A theory of force allocation can be developed based on fundamentals similar to those used for the two contact case above $\mathrm{e}^{3}$. Space does not permit detailed presentation of that solution here. However, the result is that the optimal strategy is to identify the two contacts that are best placed to provide traction and to unweight all other wheels. The contact forces at those two remaining contacts are allocated according to the two contact point solution derived above. Heuristically, traction force is developed from friction, which is proportional to normal contact force. Since normal contact force is a result of vehicle weight, or more generally the combination of weight and inertia force, it is bounded. Therefore it makes sense to concentrate that load at the contacts that are able to generate the most traction from it. Since the minimum number of contacts that can generate a given resultant is two, the optimal solution is to apply all load to the best two contacts. 


\section{DISCUSSION}

The tactic of placing all the resultant force on one side of the vehicle upon the two wheels best located to generate traction, and of unweighting all other wheels on that side, optimizes the available traction. However, on uneven terrain the choice of the two wheels best located to generate traction will change quite frequently, with resulting radical changes in wheel loading. This produces the same kind of step changes in the force commands, or "chatter" that was noted above with respect to solution by linear programming. This is hardly surprising, since both solution methods are, in principle, optimal for the planar force allocation problem. The method discussed here can be viewed as a closed form solution that is equivalent to the iterative linear programming solution.

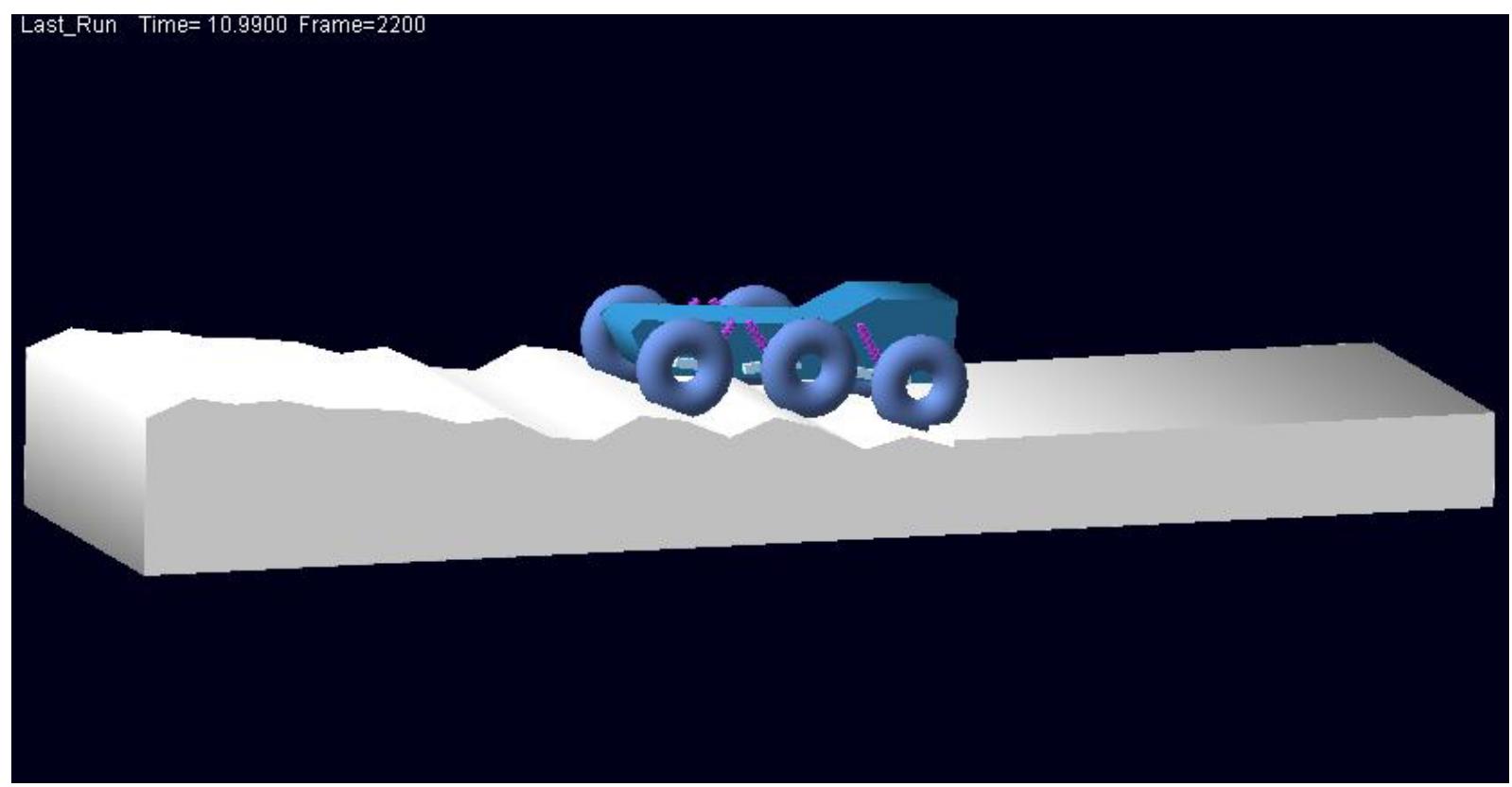

Figure 8: A frame from an ADAMS simulation of the UGCV vehicle traversing rough, random terrain. This was a test in simulation of the traction maximizing algorithm discussed in this paper.

The drastic changes in loading required by the traction maximizing solution are undesirable from several points of view. They can be de-stabilizing because of the difference between static and kinetic friction coefficients, that is not modeled in the derivation of the algorithm. Further, the large swings in commanded wheel torque and suspension load would necessarily be hard on mechanical components. Thus, we have the necessity of filtering the force commands to ease the transitions between loading regimes. That, in turn, moves the algorithm away from optimality.

We have extensively studied the behavior of the traction maximizing algorithm in simulation. Figure 8 is a frame from an ADAMS simulation of the UGCV vehicle crossing a large obstacle of random profile. As might be expected, use on moderate terrain is problematic because of relatively frequent changes in loading regimes and singularity of the solution that occurs if the terrain approximates a plane. Therefore, it is necessary to use either the zero interaction force solution, or that of Figure 5, for all but severe terrain conditions. We continue to explore the issue of when it is most appropriate to switch to the traction maximizing algorithm, and vice- 
versa. We also are seeking ways of filtering the commanded force system that will be compatible with the dynamics of the system.

\section{ACKNOWLEDGMENTS}

This research was supported by DARPA's Unmanned Ground Combat Vehicle Program and SAIC Corporation. The authors would also like to thank Mr. Raghavan Madhavan for his work in coding the proprioceptive algorithm.

\section{REFERENCES}

1. D.A. Weeks, J.H. Beno, A.M. Guenin, and D.A. Bresie, "Electromechanical Active SuspensionDemonstration for Off-Road Vehicles," Publication 2000-01-0102, SAE International Congress and Exposition, Detroit, Michigan, 2000.

2. K.J. Waldron, R.C. Arkin, D. Bakkum, E. Merrill, M. Abdallah, "Proprioceptive Control for a Robotic Vehicle Over Geometric Obstacles," to appear in Proceedings of the 2003 IEEE International Conference on Robotics and Automation, Taipei, Taiwan, May 12-17, 2003.

3. K.J. Waldron,"Near Optimal Coordination of Legged Vehicles Over Large Obstacles", Proceedings of ARK 2002, ed. Lenarcic, J. and Thomas, F., Caldes de Malavella, Spain, Kluwer, Dordrecht, pp. 501-515.

4. Hunt, K. H., 1978, Kinematic Geometry of Mechanisms, Clarendon Press, Oxford.

5. Klein, C. A. and Chung, T. S., 1987, "Force Interaction and Allocation for the Legs of a Walking Vehicle, IEEE Transactions on Robotics and Automation, Vol. RA-3, No. 6, pp. 546555.

6. Kumar, V. and Waldron, K.J. 1990, "Force Distribution in Walking Vehicles," ASME Trans. Journal of Mechanical Design, Vol. 112, 1990, pp. 90-99.

7. Pugh, D.R., Ribble E.A., Vohnout V.J., Bihari, T.E., Walliser, T.M., Patterson, M.R., Waldron, K.J., 1990, “Technical Description of the Adaptive Suspension Vehicle," International Journal of Robotics Research, Vol. 9, No. 2, pp. 24-42.

8. Kumar, V. and Waldron, K. J., 1988, "Force Distribution in Closed Kinematic Chains" IEEE Transactions on Robotics and Automation, Vol. 4, No. 6, pp. 657-664.

9. Iagnemma, K., and Dubowsky, S. "Mobile robot rough-terrain control (RTC) for planetary exploration," Proceedings of 26th Biennial Mechanisms and Robotics Conference of the 2000 ASME Design Engineering Technical Conferences, September 10-13, 2000. 